(C) 1981. The Genetical Society of Great Britain

\title{
DISEQUILIBRIUM BETWEEN LINKED INVERSIONS: AN ALTERNATIVE HYPOTHESIS
}

\author{
A. G. CLARK* and M. W. FELDMAN \\ Department of Biological Sciences, Stanford University, Stanford, CA 94305
}

Received 15.x.80

\section{SUMMARY}

\begin{abstract}
Data are presented which demonstrate a difference in the rate of recombination between cis and trans inversion double heterozygotes in Drosophila melanogaster. The corresponding two-locus theoretical model in which the double heterozygotes have different rates of recombination and no selection operates is analysed. We show that stable linkage disequilibrium can be maintained in this neutral situation. These results bear on the general observation in wild populations of several insect species that linked inversions manifest linkage disequilibrium.
\end{abstract}

\section{INTRODUCTION}

ONE of the most consistent observations in population genetics is the occurrence of linkage disequilibrium between linked inversions. Although most of these data concern Drosophila (Blight, 1955; Brncic, 1953; Krimbas, 1964; Levitan, 1955, 1953a, 1953b, 1959, 1978a, 1973b; Mather, 1963; Stalker, 1959; Toyofuku, 1962), other insect taxa also polymorphic for inversions frequently manifest disequilibrium (Landau, 1962; Martin, 1962; Rothfels and Fairlee, 1957; Lewontin and White, 1960). Classically these observations have been cited as evidence for the operation of natural selection, but direct evidence of selective differences between two-locus heterokaryotypes is lacking. The operation of natural selection is inferred from observed linkage disequilibrium in conjunction with the results of two-locus genetic theory. This theory predicts that in an infinite randomly mating population, stable linkage disequilibrium will only occur in populations under certain conditions on the relative fitnesses of genotypes. The strongest case for natural selection based solely on genotype frequencies in natural populations would be the observation of strong linkage disequilibrium between fairly loosely linked genes (or inversions) in a large panmictic closed population. Otherwise explanations which do not invoke selection cannot be discounted (see e.g., Mukai, 1977).

Selectively neutral explanations for the observation of linkage disequilibrium fall into three categories:

(1) the populations are not at equilibrium,

(2) genetic drift,

(3) population structure.

If populations are not sampled repeatedly over a long period of time, it is difficult to rule out the first possibility. A very low rate of recombination results in a slow decay of linkage disequilibrium, so the observed disequilibria could be explained by a founder effect. If the inverted sequence

\footnotetext{
* Present address: Dept. of Zoology, Arizona State University, Tempe, AZ 85281.
} 
occurred as a unique event, then it must have been in linkage disequilibrium with other sequences on the same chromosome. In the case of closely linked inversions the breakdown of linkage disequilibrium could be retarded by a very low rate of recombination due to a failure of chromosome synapsis (Lefevre, 1976). If inversion breakages occur randomly on chromosomes, then most spontaneous inversions would occur on the most common "standard" sequence and a deficit of chromosomes bearing two inversions would be expected. The data from natural populations indicate to the contrary that the standard and the doubly inverted sequences are the most common (see Discussion). Krimbas and Loukas (1980) present evidence that there are hot spots on the chromosome at which breaks are likely to occur. They give further evidence that inversions occur non-randomly by demonstrating that the distribution of inversion lengths is non-random. More data on the spontaneous rate and distribution of inversions are needed before the contribution of non-randomness in the origin of inversions (founder effects) to observed disequilibria can be assessed.

In small populations genetic drift may be a factor in determining chromosome frequencies, and hence linkage disequilibrium (Sved, 1968; Hill and Robertson, 1968). Accurate estimates of Drosophila population sizes have not been made, so that the importance of sampling effects cannot be directly evaluated. The stable maintenance of the same linkage disequilibrium throughout a species range could not be explained by drift (Baker, 1975), but there are several instances of linkage disequilibrium being reversed in sign in different areas (Levitan, 1973b; Loukas et al., 1979). Other patterns of disequilibrium that cannot be explained by drift include sex differences and seasonal cycling (Levitan, 1955, 1973a, 1973b).

Even fairly small amounts of migration between two areas of differing karyotype frequencies can produce a cline in disequilibrium (Feldman and Christiansen, 1975), so observation of such a cline (Levitan 1959) is not conclusive evidence of selection. Other aspects of population structure such as positive and negative assortative mating with respect to karyotype could generate disequilibrium, but there is no datum indicating that assortative mating is occurring in Drosophila in the field.

In this paper we present experimental data which demonstrate a different rate of recombination for cis and trans double heterokaryotypes. This motivated our theoretical analysis of a neutral two locus model with this property. It is shown that stable linkage disequilibrium can be maintained by a cis-trans recombination difference, which therefore provides another hypothesis compatible with the observation of linkage disequilibrium in field data.

\section{MATERIALS AND METHODS}

Stocks of Drosophila melanogaster bearing marked second chromosome inversions were obtained from the Bowling Green Stock Center. Flies designated $\mathrm{Cy}$ bear the Curly wing marked inversion (In(2L)22D1-2; 33F5-34A1) which is located on the left arm of chromosome II. The $\mathrm{Pm}^{2}$ chromosome (Schultz and Dobzhansky, 1934) bears the pericentric inversion (In (2LR-40F; 59E). Since one breakpoint of this inversion falls in the brown locus, flies show the Plum eye color phenotype. Because the inversions on these chromosomes do not overlap, they can recombine, and it 
is possible to construct cis $\left(\mathrm{CyPm}^{2} /++\right)$ and trans $\left(\mathrm{Cy}+/+\mathrm{Pm}^{2}\right)$ double heterozygotes. The stocks designated as heterogeneous background were intercrossed for 7 generations, while the homogeneous background stocks were made isogenic for the $\mathrm{X}$ and part of the second chromosome (see Clark and Feldman, 1981).

Recombination test crosses were performed with cis and trans double heterozygote females designated respectively as the cis cross and the trans cross. Both genetic backgrounds were employed separately in the test crosses, and wild phenotype males of the respective background were used. Four day old virgins of appropriate genotypes were placed as pairs in $95 \mathrm{~mm}$ shell vials with $2 \mathrm{~g}$ of Carolina 4-24 instant Drosophila medium, $5 \mathrm{ml}$ of water and live yeast. After 4 days of egg laying, adults were removed. On the eighteenth day the adult progeny were scored by phenotype, and the ratio of these phenotypes was used to estimate the rate of recombination. Cultures were maintained in a controlled light and temperature incubator at $25 \pm 1^{\circ} \mathrm{C}$ with a 12 hour photoperiod.

To obtain estimates of the recombination rates in the cis and trans crosses independently, the phenotype frequencies must be weighted by their viabilities (Muller, 1916). If we designate the viabilities as $v_{1}$ for $\mathrm{Cy}, v_{2}$ for $\mathrm{Pm}^{2}, v_{3}$ for CyPm2, and $v_{4}$ for wild type, then the cis and trans estimates for the rate of recombination are:

$$
R=\frac{\left(f_{1} / v_{1}\right)+\left(f_{2} / v_{2}\right)}{\sum_{i}\left(f_{i} / v_{i}\right)} \quad r=\frac{\left(f_{3} / v_{3}\right)+\left(f_{4} / v_{4}\right)}{\sum_{i}\left(f_{i} / v_{i}\right)}
$$

where $R$ represents the recombination rate in the cis cross, and $r$ represents the recombination rate in the trans cross. The viabilities were estimated using the experimental procedure outlined in Clark and Feldman (1981) and are reported in table 1. Distributions for the estimates of $r$ and $R$ were

\section{TABLE 1}

Estimates of egg-to-adult viability relative to wild type based on deviations from Mendelian segregation and their empirical standard errors. Numbers in parentheses indicate sample sizes (number of progeny sets, number of progeny scored)

Heterogeneous background

$$
\begin{array}{cccc}
\text { Cy } & & \mathrm{Cy}+ & \mathrm{CyPm}^{2} \\
+\mathrm{Pm}^{2} & +\mathrm{Pm}^{2} & \frac{\mathrm{C}^{2}}{+} \\
0.855 \pm 0.013 & 0.968 \pm 0 \cdot 020 & 0 \cdot 900 \pm 0 \cdot 054 & 0 \cdot 950 \pm 0 \cdot 021 \\
(391,31475) & (329,25138) & (42,1911) & (264,15683)
\end{array}
$$

Homogeneous background

$\begin{array}{cccc} & & \mathrm{Cy}+ & \mathrm{CyPm}^{2} \\ \mathrm{Cy} & \mathrm{Pm}^{2} & \frac{\mathrm{Pm}^{2}}{+} & \frac{0.774 \pm 0 \cdot 031}{+} \\ 0.908 \pm 0 \cdot 013 & 0.998 \pm 0 \cdot 029 & 0.875 \pm 0.038 \\ (440,33008) & (270,16312) & (100,4968) & (132,9298)\end{array}$

plotted, and the Kolmogorov-Smirnov test verified that they were distributed approximately normally. Standard errors were calculated empirically from the distributions, and were used in parametric comparisons. 


\section{Results}

\section{(i) Data analysis}

Histograms of the recombination fraction in stocks with heterogeneous background are presented in fig. 1. Because the variance in $r$ is sufficiently greater than the binomial expectation, the frequencies are not weighted by the number of progeny per female. This reduces the bias in the estimate of the variance (Engels, 1979). Each unit on the $\mathrm{Y}$ axis is therefore a single
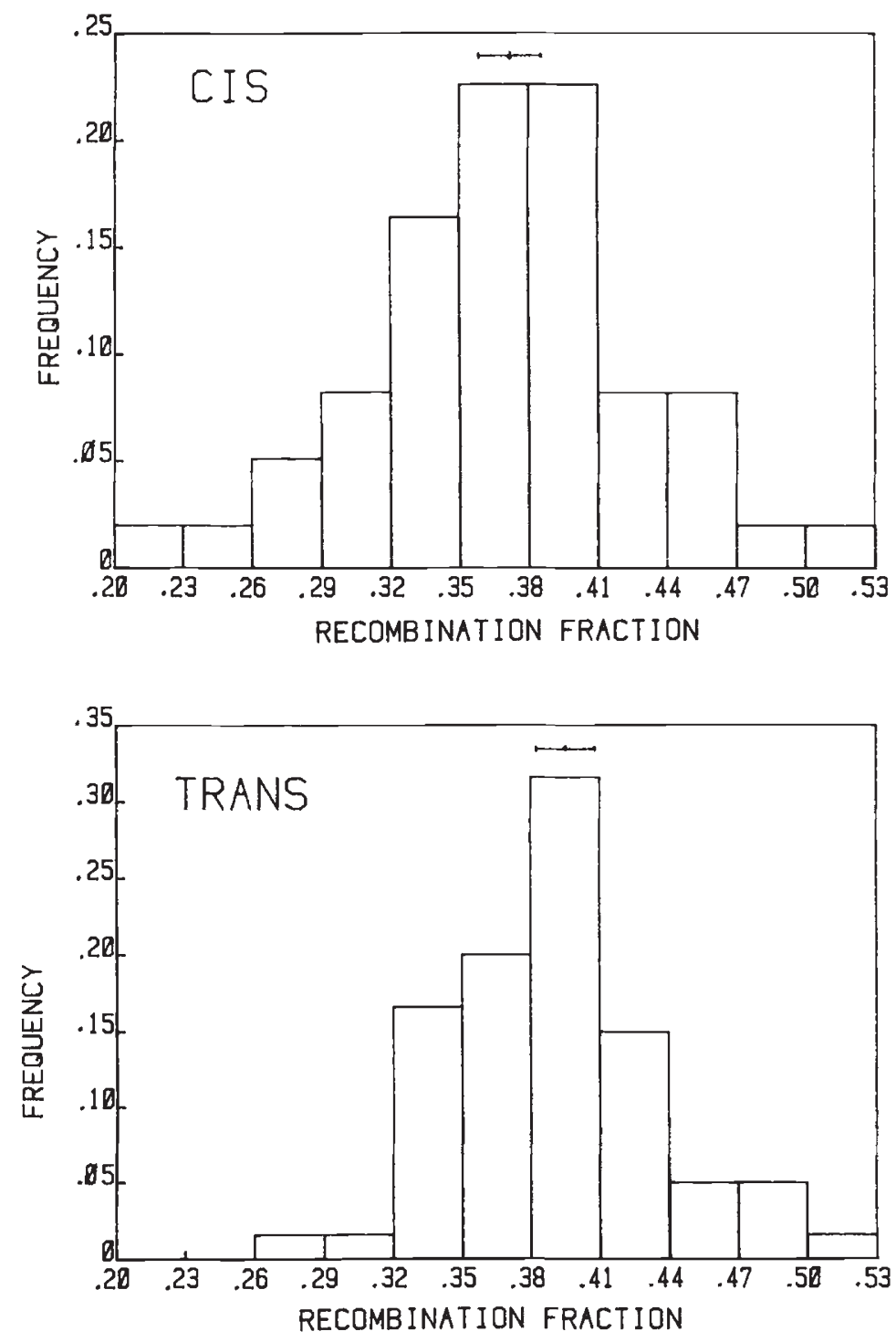

FIG. 1.-Histograms of recombination rate in cis and trans crosses among flies with a heterogeneous background. Ninety-five per cent confidence intervals on the means appear on the graphs and they are: $R=0.373 \pm 0 \cdot 012, r=0.395 \pm 0 \cdot 013$. Sample sizes are: $c i s-96$ crosses and 7452 progeny, trans -60 crosses and 5003 progeny. 
progeny set. The variances of $r$ and $R$ are not different by the $\mathrm{F}$ test, and analysis of variance indicates a significant difference between the mean recombination rates among females of different chromosome phase (table 2).

TABLE 2

Results of the analysis of variance for comparisons of mean recombination fraction between cis and trans crosses. Distributions being compared appear in figs. 1 and 2

Heterogeneous background

$\begin{array}{lrcccc}\text { Source } & \text { d.f. } & \text { SS } & \text { MS } & \text { F } & \\ \text { Among } & 1 & 0.016 & 0.016 & 5.41 & P<0.025 \\ \text { Within } & 154 & 0.466 & 0.003 & & \\ \text { Total } & 155 & 0.483 & & & \end{array}$

Homogeneous background

$\begin{array}{lrcccc}\text { Source } & \text { d.f. } & \text { SS } & \text { MS } & \text { F } & \\ \text { Among } & 1 & 0.135 & 0.135 & 49.43 & P<0.001 \\ \text { Within } & 171 & 0.465 & 0.003 & & \\ \text { Total } & 172 & 0.600 & & & \end{array}$

The cis recombination rate is lower than the trans recombination rate in stocks with homogeneous background as well (fig. 2). Both of these results are quite robust with respect to weighting. If phenotype frequencies are not weighted by viabilities, then in the heterogeneous background $R=$ $0 \cdot 356 \pm 0.006$, and $r=0.406 \pm 0.006$, and with the homogeneous background $R=0.357 \pm 0.006$ and $r=0.395 \pm 0.005$. In both cases we still have $R<r$. Standard errors are changed slightly if the recombination frequencies are weighted by the size of each progeny set, but the significance of the difference between $R$ and $r$ remains unchanged.

\section{(ii) Theoretical analysis}

Models of the evolution of two loci normally assume an equivalence of double heterozygotes in fitness and rate of recombination. The observation of a position effect in recombination leads us to relax this assumption, and to consider a model in which no selection or migration occurs, but where crossover rates depend on double heterozygote phase. The model assumes random mating in an infinite population with genotypes identified by two diallelic loci. The only factors changing the chromosome frequencies are therefore segregation and recombination.

Let the alleles at one locus be identified as $A$ and $a$, and the alleles at the other locus are $B$ and $b$. The chromosome types $A B, A b, a B$, and $a b$ have frequencies $x_{1}, x_{2}, x_{3}$, and $x_{4}$ respectively. The rate of recombination in the cis double heterozygotes is $R$, while in the trans double heterozygotes it is $r$. Unlike the situation in Drosophila, we will assume that both sexes have an equal rate of recombination. The recursion of chromosome frequencies is then:

$$
\begin{aligned}
& x_{1}^{\prime}=x_{1}-R x_{1} x_{4}+r x_{2} x_{3}=x_{1}-D^{*} \\
& x_{2}^{\prime}=x_{2}+R x_{1} x_{4}-r x_{2} x_{3}=x_{2}+D^{*} \\
& x_{3}^{\prime}=x_{3}+R x_{1} x_{4}-r x_{2} x_{3}=x_{3}+D^{*} \\
& x_{4}^{\prime}=x_{4}-R x_{1} x_{4}+r x_{2} x_{3}=x_{4}-D^{*}
\end{aligned}
$$



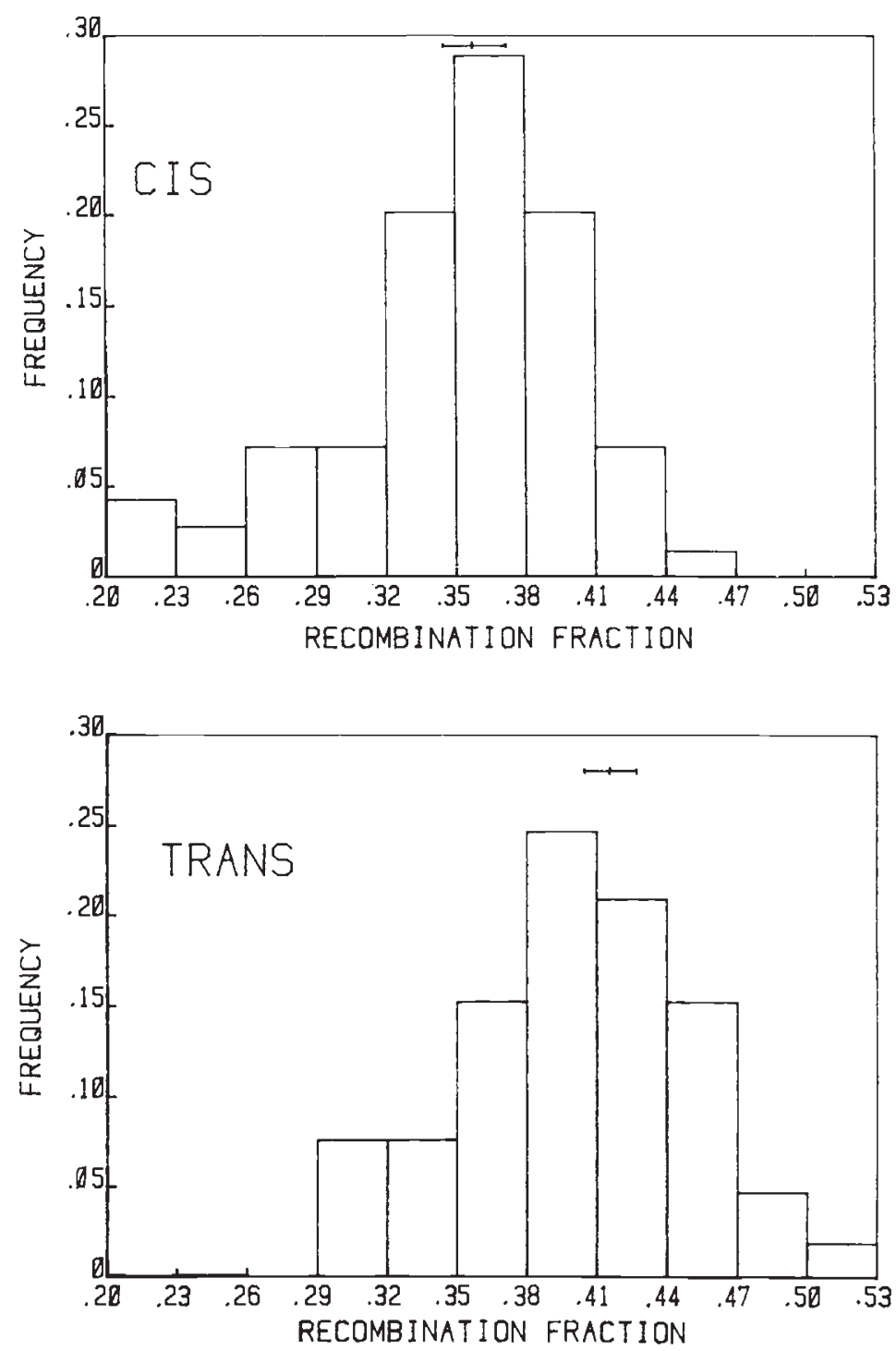

FIG. 2.-Histograms of recombination rate in cis and trans crosses among flies with homogeneous background. Ninety-five per cent confidence intervals are: $\boldsymbol{R}=0 \cdot 357 \pm 0.012$, $r=0.414 \pm 0.010$. Sample sizes are: cis -68 crosses and 7773 progeny, trans -105 crosses and 10,047 progeny.

where $D^{*}=R x_{1} x_{4}-r x_{2} x_{3}$. This system of equations is analogous to the no selection case with one recombination value, where $r D$ is replaced by $D^{*}$. If $R=r$, then $D^{*}=r D$ and this quantity decays geometrically to zero at rate $(1-r)$ per generation. From equation (1), the dynamic of $D^{*}$ is determined by:

$$
\begin{aligned}
D^{* \prime} & =R\left(x_{1}-D^{*}\right)\left(x_{4}-D^{*}\right)-r\left(x_{2}+D^{*}\right)\left(x_{3}+D^{*}\right) \\
& =D^{*}\left[1-R\left(x_{1}+x_{4}\right)-r\left(x_{2}+x_{3}\right)+D^{*}(R-r)\right] .
\end{aligned}
$$


The quantity in brackets is less than one because $\left(x_{1}+x_{4}\right)>\left(x_{1} x_{4}\right)$ and $\left(x_{2}+x_{3}\right)>\left(x_{2} x_{3}\right)$ and $(R-r)<1$. $D^{*}$ therefore decays to zero at a rate asymptotically dependent on the average of the recombination rates weighted by the appropriate combinations of gene frequencies.

At equilibrium, we have $D^{*}=0$, so that

$$
\left(\hat{x}_{1} \hat{x}_{4}\right) /\left(\hat{x}_{2} \hat{x}_{3}\right)=r / R \text {. }
$$

From equation (1) the allele frequencies remain constant over time. Let $\mathrm{A}, \mathrm{a}, \mathrm{B}$ and $\mathrm{b}$ represent the frequencies of alleles $A, a, B$ and $b$ respectively. At equilibrium $D^{*}=0$, which can be rewritten,

$$
R(\mathrm{AB}+\hat{D})(\mathrm{ab}+\hat{D})=r(\mathrm{Ab}-\hat{D})(\mathrm{aB}-\hat{D}) .
$$

The equilibrium value $\hat{D}$ of the linkage disequilibrium is therefore the root of the quadratic,

$$
0=D^{2}[1-(r / R)]+D[(\mathrm{AB}+\mathrm{ab})+(r / R)(\mathrm{Ab}+\mathrm{aB})]+[1-(r / R)](\mathrm{ABab}) .
$$

If $R>r$ then the only valid root of this quadratic is negative, while if $R<r$ then $D$ is positive.

To prove that there is only one valid root, observe that,

$$
D(\max )=\min (\mathrm{Ab}, \mathrm{aB}) \text {. }
$$

Substituting $D=\mathrm{Ab}$ into (5), we get,

$$
\begin{aligned}
0 & =\mathrm{A}^{2} \mathrm{~b}^{2}[1-(r / R)]+\mathrm{Ab}[\mathrm{AB}+\mathrm{ab}+(r / R)(\mathrm{Ab}+\mathrm{aB})]+[1-(r / R)](\mathrm{ABab}) \\
& =\mathrm{A}^{2} \mathrm{~b}^{2}+\mathrm{A}^{2} \mathrm{Bb}+\mathrm{Aab}^{2}+\mathrm{ABab} .
\end{aligned}
$$

The value of the quadratic is positive at $D(\max )$ and negative at $\hat{D}=0$, so there can be only one valid root. By symmetry, if $r<R$ there is a unique valid root with $D<0$. In summary, allele frequencies do not change in this model, and $D^{*}$ tends to zero. The unique stable equilibrium value of the linkage disequilibrium depends on the initial allele frequencies and the ratio $r / R$ (see fig. 3). A numerical example of the trajectories of $D$ and $D^{*}$ is presented in fig. 4.

In many organisms, including Drosophila, the two sexes have different rates of recombination. If in females $R$ is the cis rate of recombination, and $r$ is the trans rate, and in males $S$ and $s$ are the respective crossover frequencies, then we can define,

$$
D^{*}(f)=R x_{1} x_{4}-r x_{2} x_{3}, \quad D^{*}(m)=S x_{1} x_{4}-s x_{2} x_{3} .
$$

Both sexes contribute gametes equally to both sexes in the progeny, so after one generation the chromosome frequencies are the same in both sexes. If we let $x_{i}(f)$ and $x_{i}(m)$ be the initial chromosome frequencies in females and males respectively, then

$$
\begin{aligned}
& T x_{1}^{\prime}=x_{1}(f)-D^{*}(f)+x_{1}(m)-D^{*}(m) \\
& T x_{2}^{\prime}=x_{2}(f)+D^{*}(f)+x_{2}(m)+D^{*}(m) \\
& T x_{3}^{\prime}=x_{3}(f)+D^{*}(f)+x_{3}(m)+D^{*}(m) \\
& T x_{4}^{\prime}=x_{4}(f)-D^{*}(f)+x_{4}(m)-D^{*}(m)
\end{aligned}
$$




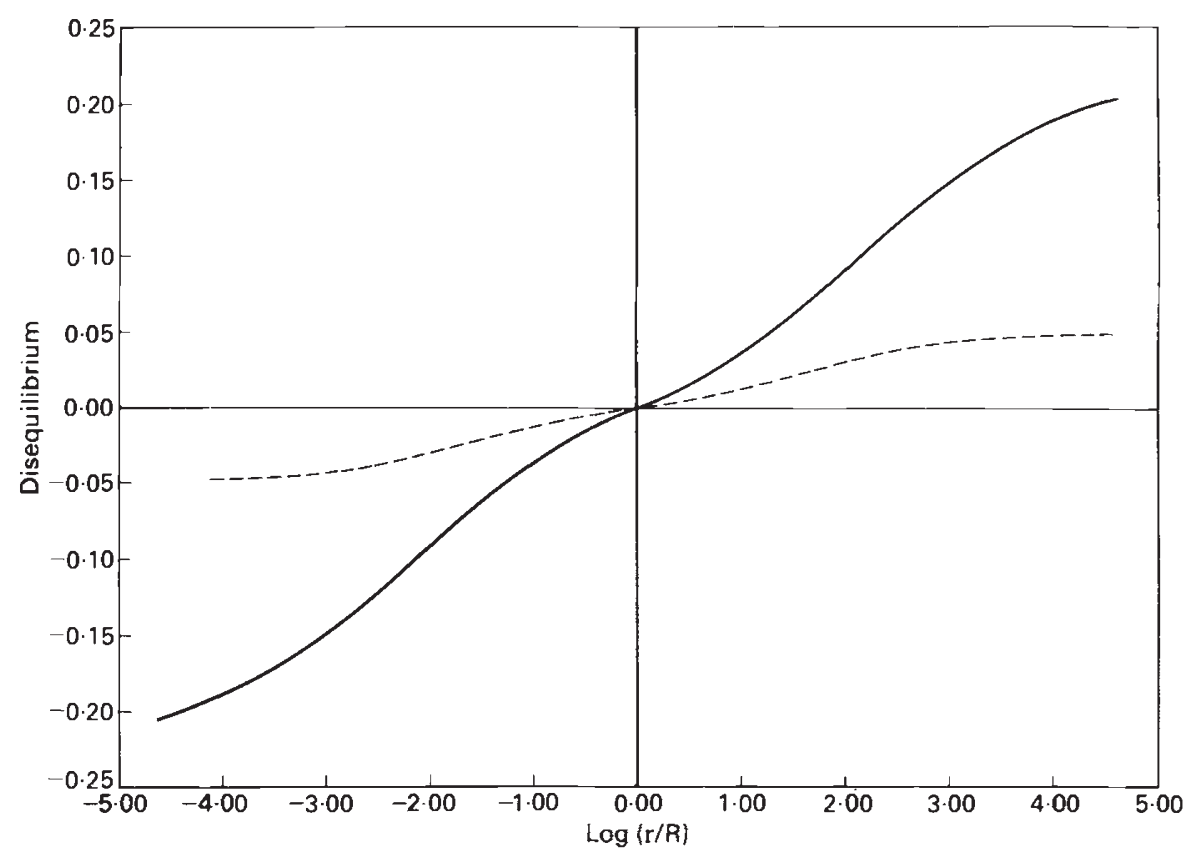

FIG. 3.-Relation between $\log (r / R)$ and $D$ for two sets of allele frequencies. The solid line represents all alleles equally frequent, while the dashed line represents the case $p(A)=0 \cdot 9$, $p(B)=0 \cdot 5$.

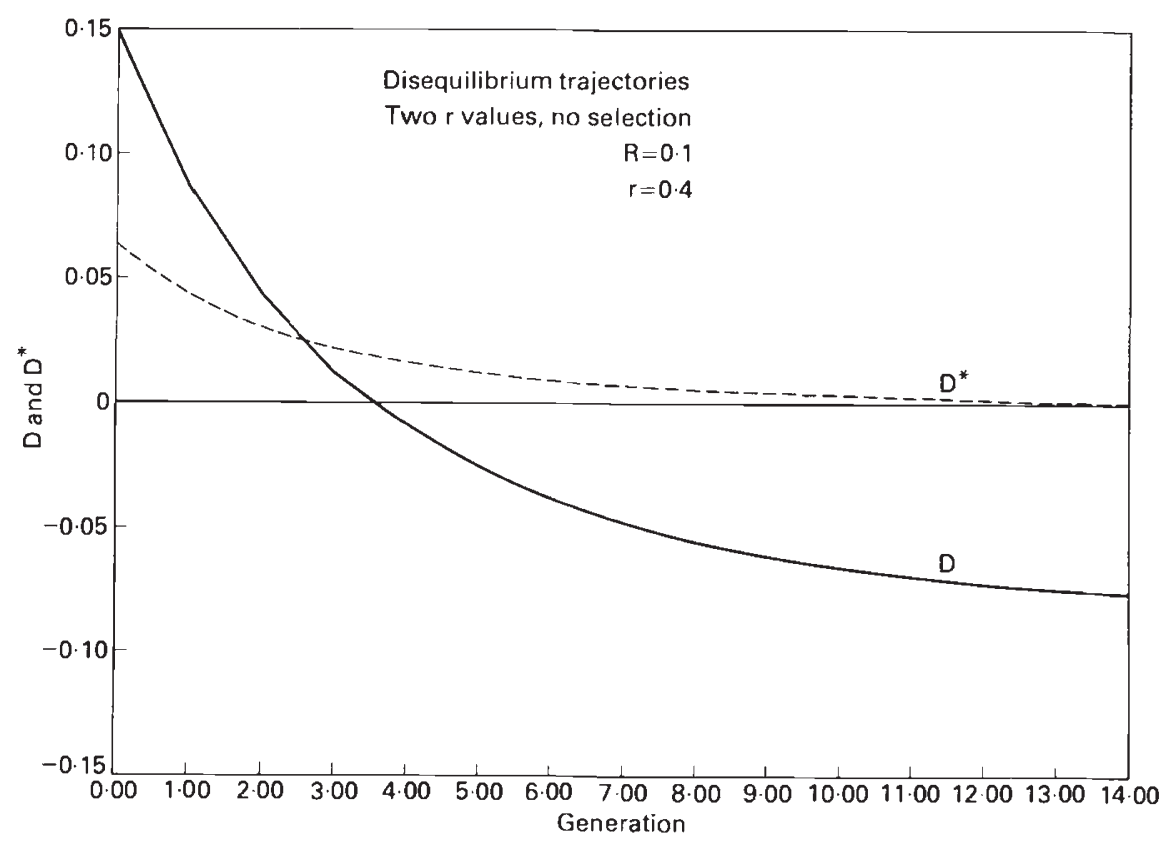

FIG. 4.-Numerical example of trajectories of $D$ and $D^{*}$ with no selection. Initial chromosome frequencies are $x 1=x 4=0 \cdot 4, x 2=3=0 \cdot 1$. 
where $T$ normalizes the frequencies. Since after the first generation $x_{i}(f)=$ $x_{i}(m)$ these equations can be rewritten,

$$
\begin{aligned}
& T x_{1}^{\prime}=x_{1}-\left[D^{*}(f)+D^{*}(m)\right] / 2 \\
& T x_{2}^{\prime}=x_{2}+\left[D^{*}(f)+D^{*}(m)\right] / 2 \\
& T x_{3}^{\prime}=x_{3}+\left[D^{*}(f)+D^{*}(m)\right] / 2 \\
& T x_{4}^{\prime}=x_{4}-\left[D^{*}(f)+D^{*}(m)\right] / 2 .
\end{aligned}
$$

If we define $D^{* *}=\left[D^{*}(f)+D^{*}(m)\right] / 2$, then the recursions are identical to the one sex case with $D^{*}$ replaced by $D^{* *}$. $D^{* *}$ can be written,

$$
D^{* *}=[(R+S) / 2] x_{1} x_{4}-[(r+s) / 2] x_{2} x_{3}
$$

so the two sex case is analogous to the one sex case except the cis and trans recombination rates are averaged over sexes. This is similar to the two sex case with $R=r$ and $S=s$ (Geiringer, 1948). In the two sex case, $D^{* *}$ approaches zero and the equilibrium value of $D$ depends on [1$(r+s) /(R+S)]$ and the allele frequencies. Allele frequencies are the same in both sexes after the first generation, and the equilibrium value of $D$ is the valid root of (5) with allele frequencies equal to the average over sexes of the initial allele frequencies. Note that if $S=s=0$, then $D$ is the same as the one sex case with recombination values determined by the female.

If inversions differ in their selective values, then only under certain conditions will linkage disequilibrium be expected. In particular, in the additive fitness case (Bodmer and Felsenstein, 1967), stable linkage disequilibrium is never achieved. This situation is changed if the cis and trans double heterozygotes have different recombination rates. In the appendix, we show that stable linkage disequilibrium can be maintained with additive fitnesses if there is a phase difference in the crossover rate, and we briefly consider the multiplicative fitness model.

\section{Discussion}

From the theory we have developed, cis-trans differences in rates of recombination can lead to stable linkage disequilibrium. Our observation of cis-trans differences in laboratory Drosophila melanogaster leads us to ask whether this work has relevance to the observation of linkage disequilibrium between linked inversions. A direct answer would come from linkage tests to determine rates of crossover between inversion breakpoints. Unfortunately it is a very laborious task to do linkage studies when progeny phenotypes are polytene chromosome banding patterns, and they have been done in only a few cases (Levitan, 1973b). Absolute linkage disequilibrium between inversions and electrophoretic marker could be used to facilitate the linkage tests, but the procedure is still considerably more difficult than when dominant markers conveniently identify the inversions. Observation of chiasma frequency can give an indication of the rate of crossover, and these studies generally indicate a very low rate of crossover in double inversion bearing chromosomes (Krimbas and Loukas, 1980). If the recombination values are low, then small differences between them will result in a larger change in the ratio $r / R$, and it is in this sense "easier" to 
generate disequilibrium. With very tight linkage it takes many generations for disequilibrium to decay, so founder effects and drift may also play a bigger role. Virtually all two locus models predict that higher values of linkage disequilibrium are more likely with tighter linkage, so a particular relation between $r$ and $D$ cannot be used to assess the relevance of different models. With the exception of Lewontin and White (1960), unlinked inversions are generally uncorrelated. This however is not sufficient grounds to rule out selection as a significant force, because it takes strong epistasis to maintain disequilibrium between unlinked genes. On the other hand, we cannot conclude that tight linkage alone is sufficient to explain the observed disequilibria.

The theory predicts that equilibrium populations will have an excess of chromosomes of the double heterozygote with the lower rate of recombination. We found that $R<r$, and would therefore predict that there would be an excess of non-inverted and doubly inverted chromosomes. In at least 12 populations this has been the direction of the observed disequilibrium (Levitan, 1955, 1973b; Brncic, 1961; Stalker, 1964; Martin, 1962). Only one exception to this pattern has been found (Levitan, 1973b), and these same inversions occur in linkage disequilibrium in the opposite direction in another population.

Patterns of disequilibrium may yield information about their cause. Levitan $(1973 a, 1973 b)$ observed elevation gradients, seasonal variation, and sex differences in the magnitude of linkage disequilibrium. Clines may be due to selective differences in different environments, or migration between populations possessing different chromosome frequencies. Seasonal variation in $D$ could be caused by seasonal variation in the frequency of 1 chromosome, which may again be caused by selection or migration. Sex differences may also be due to selection or sex-specific migration. Cis-trans differences in the rate of recombination by itself will not generate these patterns, but in conjunction with other forces it could. Existence of these patterns does not therefore close the possibility that cis-trans differences may be a causative factor in these disequilibria.

It should be noted that not all linked inversions are in linkage disequilibrium, but this does not contradict our theory. We have no reason to expect that all pairs of linked inversions should have cis-trans differences in the rates of recombination. We only mean to suggest that in inversion pairs with cis-trans differences, linkage disequilibrium can be generated in the absence of selection. In addition, even if there is a cis-trans difference, the theory predicts that the observed disequilibrium may be small. There is not a unique mapping from $(r / R)$ to $D$, because the value of $D$ at equilibrium depends on the initial allele frequencies.

In conclusion, although we do not know the generality of cis-trans recombination rate differences in double inversion heterokaryotypes, it remains an open possibility that this phenomenon may be relevant to the general observation of disequilibrium between linked inversions. We also raise the more general theoretical question of the importance of cis-trans differences in the evolution of recombination rates. 


\section{REFERENCES}

BAKER, W. K. 1975. Linkage disequilibrium over space and time in natural populations of Drosophila montana. Proc. Nat. Acad. Sci., 72, 4095-4099.

BLIGHT, W. C. 1955. A cytological study of linear populations of Drosophila americana near St. Louis, Mo. Ph.D. thesis, Washington University, St. Louis.

BODMER, W. F., AND FELSENSTEIN, J. 1967. Linkage and selection: Theoretical analysis of the deterministic two locus random mating model. Genetics, 57, 237-265.

BRNCIC, D. 1953. Chromosomal variation in natural populations of D. guaramunu. Z. Ind. Abst. Vererb., 85,1-11; 1961. Nonrandom association of inversions in Drosophila pavani. Genetics, 46, 401-406.

CLARK, A. G., AND FELdMAN, M. W. 1981. The estimation of epistasis in experimental populations of Drosophila melanogaster. II. Assessment of meiotic drive, viability, fecundity and sexual selection. Heredity, 51-81.

ENGELS, w. R. 1979. The estimation of mutation rates when premeiotic events are involved. Envir. Mutagenesis, 1, 37-43.

FELDMAN, M. W., AND CHRISTIANSEN, F. B. 1975. The effect of population subdivision on two loci without selection. Genet. Res., 24, 151-162.

GEIRINGER, H. 1948. On the mathematics of random mating in case of different recombination values for males and females. Genetics, 33, 548-564.

HILL, W. G., AND ROBERTSON, A. 1968. Linkage disequilibrium in finite populations. Theor. Appl. Genetics, 38, 226-231.

KRIMBAS, C. B. 1964. The genetics of Drosophila subobscura populations. II. Inversion polymorphism in a population from Holland. Z. Vererb., 95, 125-128.

KRIMBAS, C. B., AND LOUKAS, M. 1980. The inversion polymorphism of Drosophila subobscura, Evol. Biol., 12, 165-234.

LANDAU, R. 1962. Four forms of Simulium tuberosum (Lunstr.) in Southern Ontario: a salivary gland chromosome study. Can. J. Zool., 40, 921-939.

LEFEVRE, G. 1976. A photographic representation of the polytene chromosomes of Drosophila melanogaster salivary glands. In The Genetics and Biology of Drosophila, Vol. 1a, eds. M. Ashburner and E. Novitski. Academic Press, New York.

LEVITAN, M. 1955. Studies of linkage in populations. I. Associations of second chromosome inversions in Drosophila robusta. Evolution, 9, 62-74; 1958a. Studies of linkage in populations. II. Recombination between linked inversions of D. robusta. Genetics, 43 , 620-633; 1958b. Non-random associations of inversions. Cold Spring Harbor Symp. Quant. Biol., 23, 251-268; 1959. A non-random association of inversions correlated with their geographic distribution. Genetics, 44, 522; 1973a. Studies of linkage in populations. VI. Periodic selection for X chromosome gene arrangement combinations. Evolution, 27, $215-232 ; 1973 b$. Studies of linkage in populations. VII. Temporal variation and X chromosome linkage disequilibrium. Evolution, 27, 476-485.

LEWONTIN, R. C., AND WHITE, M. J. D. 1960. Interaction between inversion polymorphisms of two chromosome pairs in the grasshopper Moraba scurra. Evolution, 14, 116-129.

LOUKAS, M., KRIMBAS, C. B., AND VERGINI, Y. 1979. The genetics of Drosophila subobscura. IX. Studies on linkage disequilibrium in four natural populations. Genetics, 93, 497-523.

MARTIN, J. 1962. Interrelation of inversion systems in the midge Chironomus intertinctus. I. A sex-linked inversion. Aust. J. Biol. Sci., 15, 666-673.

MATHER, W. B. 1963. Patterns of chromosomal polymorphism in Drosophila rubida. Am. Naturalist, 97, 59-64.

Moran, P. A. P. 1967. Unsolved problems in evolutionary theory. Proc. Fifth Berkeley Symp. on Math. Stat. and Prob., Vol. IV, pp. 457-480.

MUKAI, T. 1977. Lack of experimental evidence supporting selection for the maintenance of isozyme polymorphisms in Drosophila melanogaster. Proc. Sec. Taniguchi Int. Symp. on Biophys.: Mol. Evol. and Polymorphism, ed. M. Kimura, pp. 103-126.

MULler, H. J. 1916. The mechanism of crossing-over. Amer. Naturalist 50, 350-366.

ROTHFELS, K. H., AND FAIRLEE, W. 1957. The non-random distribution of inversion breaks in the midge Tendipes decorus. Can. J. Zool., 35, 221-263.

SCHULTZ, J., AND DOBZHANSKY, TH. 1934. The relation of a dominant eye color in Drosophila melanogaster to the associated chromosomal rearrangement. Genetics, 19 , 344-364.

STALKER, H. D. 1959. Nonrandom associations of inversions in chromosomes of wild Drosophila paramelanica. Genetics, 44, 538; 1964. Chromosomal polymorphism in Drosophila euronotus. Genetics, 49, 669-687. 
SVED, J. A. 1968. The stability of linked systems of loci with small population size. Genetics, $59,543-563$.

TOYOFUKU, Y. 1962. Drosophila survey of Hokkaido. XVII. A study of chromosomal polymorphism in natural populations of Drosophila nigromaculata. Japan. J. Genet., 37, 291-301.

\section{APPENDIX}

\section{$A$ remark on selection and phase dependent recombination}

We have seen that in the absence of selection, the ultimate neutral equilibrium has $D^{*}=0$. There are three well studied selection models which, under the usual assumption $R=r>0$ have a polymorphic equilibrium with $\hat{D}=0$; these are the additive, multiplicative and symmetric viability schemes. We will show that a phase difference in the recombination rates affects this result. Using the same ordering of chromosomes as above, consider first the additive viability model (Bodmer and Felsenstein, 1967) whose selection matrix can be written as,

$$
\begin{gathered}
B B \\
A A \\
A a \\
a a
\end{gathered}\left[\begin{array}{ccc}
a_{1}+b_{1} & a_{1}+b_{2} & a_{1}+b_{3} \\
a_{2}+b_{1} & a_{2}+b_{2} & a_{2}+b_{3} \\
a_{3}+b_{1} & a_{3}+b_{2} & a_{3}+b_{3}
\end{array}\right] .
$$

The usual recursion relations for the chromosome frequencies can be used except that $D^{*}$ is substituted for $D$. Thus, for example,

$$
W x_{1}^{\prime}=x_{1}\left[a_{1} p(A)+a_{2} p(a)+b_{1} p(B)+b_{2} p(b)\right]-\left(a_{2}+b_{2}\right)\left(R x_{1} x_{4}-r x_{2} x_{3}\right)
$$

where $W$ is the mean fitness, and $p(A)$ is the frequency of the $A$ allele. The argument of Moran (1967) can be used to show that a polymorphic equilibrium (with $R \neq r$ and both positive) must have $D^{*}=0$. As we saw above, this gives a quadratic equation for $D$ in terms of the gene frequencies, and the resulting $\hat{D} \neq 0$. It is then easy to see that the gene frequencies at equilibrium are,

$$
\hat{p}(A)=\left(a_{2}-a_{3}\right) /\left(2 a_{2}-a_{1}-a_{3}\right), \quad \hat{p}(B)=\left(b_{2}-b_{3}\right) /\left(2 b_{2}-b_{1}-b_{3}\right) .
$$

These frequencies together with $\hat{D}$, the unique valid root of the quadratic in $D$ obtained by using the equilibrium gene frequencies in $D^{*}=0$ specify the single interior equilibrium. It can then be shown to be locally stable under the usual overdominance conditions, $a_{2}>a_{1}, a_{3}$ and $b_{2}>b_{1}, b_{3}$.

Recall that in the multiplicative viability model obtained from the additive case by using products instead of sums to compose the viabilities, with equal recombination rates the $\hat{D}=0$ point with gene frequencies $p(A)$, $p(B)$ is an equilibrium. The interesting fact here is that a $D^{*}=0$ equilibrium (with $\hat{D} \neq 0$ ) cannot have the gene frequencies $\hat{p}(A)$ and $\hat{p}(B)$. This can be seen at once by comparing the marginal fitnesses of the chromosomes at $D^{*}=0$. 